\title{
A new recycling method to generate turbulent inflow profiles
}

\author{
Anirban Garai*, Scott M. Murman ${ }^{\dagger}$, and Dirk Ekelschot ${ }^{\ddagger}$ \\ NASA Ames Research Center, Moffett Field, CA, USA
}

\begin{abstract}
The accuracy of the scale-resolving simulations for practical geometries strongly depends on the inflow boundary conditions. Imposing experimentally observed turbulent inflow profiles for the numerical simulations is a major challenge. Existing methods available in the literature assume self-similar behavior, which is not true for most of the experiments. In the present work, we formulate the turbulent inflow profile generation technique as an optimization problem. An adjoint technique is exploited to evaluate the sensitivities of multiple input parameters for the present problem. The present formulation is then tested to generate a laminar boundary layer profile, turbulent boundary layer profile, and turbulent jet profile.
\end{abstract}

\section{Introduction}

Scale-resolving simulations (e.g. direct numerical simulation (DNS), large-eddy simulation (LES)) of practical geometries are becoming possible with the advancement of computing resources. The accuracy of these simulations depends partly upon the time-dependent inflow, outflow, and farfield boundary conditions. Previous work examined the benefits of a perfectly matched layer (PML) approach for outflow and farfield sponge layers in turbulent flow simulations. ${ }^{1}$ The current work focuses on the inflow/upstream conditions, which directly impact the downstream turbulent flow characteristics, (e.g. transition location, boundary layer separation, etc.).

Many different techniques are available for generating turbulent inflow conditions, though most focus on incompressible flow, whereas we are interested in compressible simulations. One approach is to simulate the full natural transition process,${ }^{2}$ however this requires a long upstream region that includes the complete geometry definition (e.g. the turns in $3 \mathrm{~d}$ ducts for jet flow simulations). Further, the laminar flow must be perturbed to initiate the transition process, for example using linear stability theory, parabolized stability theory, or blowing-suction strips. ${ }^{3,4}$ At practical conditions, simulating the transition process itself is prohibitively costly.

A less expensive method is to impose the experimentally observed mean profile with some random perturbations at the inflow. ${ }^{5}$ The imposed conditions may not satisfy the compressible Navier-Stokes equations, and the approach requires a "development", or "relaxation", region where the error introduced with the manufactured inflow condition decreases. The amplitude of the synthetic turbulence can be constrained by the Reynolds stress tensor, but specification of the phase relationships for the turbulent fluctuations is extremely difficult. As the associated transition process is completely numerical, it is very difficult to extrapolate the flow parameters upstream such that the process generates the desired profile.

One of the most popular methods is to perform an auxiliary simulation to generate inflow turbulence data, and the resulting profiles are fed into the main computational domain. For example, previous work by the authors utilized homogeneous isotropic turbulence (HIT) to initiate bypass transition in DNS of a high-pressure turbine blade. ${ }^{6}$ Lund $^{7}$ devised a method to simulate a spatially developing boundarylayer by coupling the inflow with the outflow plane of the auxiliary simulation through the recycling and rescaling method. In this process, the mean and turbulent fluctuations of different state variables are

*Science and Technology Corporation, Anirban.Garai@nasa.gov

†NASA Advanced Supercomputing Division. Scott.M.Murman@nasa.gov

${ }^{\ddagger}$ Universities Space Research Association. dirk.ekelschot@nasa.gov 
scaled individually. The rescaling process is challenging to implement in a distributed parallel environment, especially using a discontinuous solution space as we desire, and extension to compressible flows is problematic (c.f. Xu \& Martin $\left.{ }^{8}\right)$.

Spalart ${ }^{9}$ developed a clever method to account for the spatial growth of the boundary layer in simulations with periodic streamwise boundary conditions by adding source terms to the Navier-Stokes equations. This introduces a coordinate transformation that minimizes the streamwise inhomogeneity. The resulting transformed equations require external inputs for the streamwise gradients. These so-called "growth terms" are complicated in form.

In the present work, we apply a source term technique, similar to Spalart's, to the compressible NavierStokes equations with periodic streamwise boundary conditions to simulate spatially developing flow profiles. By supplying appropriate source terms with periodic boundary conditions, one can simulate different instances of any spatially evolving turbulent profile, while the periodic recycling naturally generates physical turbulent fluctuations. These source terms can be computed either analytically for self-similar profiles, or by applying an adjoint-driven optimization method. The adjoint-driven optimization method has the potential to be applied to general (non self-similar) conditions.

The remainder of the paper is organized as follows. First, we will describe the computation of the source terms, both analytically and using the adjoint-optimization technique. Next we will present results for the laminar Blasius profile, a zero-pressure-gradient (ZPG) turbulent boundary-layer and a turbulent jet profile. Then, we will draw some concluding remarks and discuss the future outlook.

\section{Theory}

In this section, we will explain the basic idea behind the application of the source terms to generate spatially evolving inflow profiles in a streamwise periodic domain. The Navier-Stokes equations for compressible flow are as follows:

$$
\begin{gathered}
\frac{\partial \rho}{\partial t}+\frac{\partial}{\partial x_{j}}\left(\rho u_{j}\right)=S_{\rho}, \\
\frac{\partial\left(\rho u_{i}\right)}{\partial t}+\frac{\partial}{\partial x_{j}}\left(u_{j} \rho u_{i}\right)=-\frac{\partial p}{\partial x_{i}}+\frac{\partial \sigma_{i j}}{\partial x_{j}}+S_{\rho u_{i}}, \\
\frac{\partial(\rho E)}{\partial t}+\frac{\partial}{\partial x_{j}}\left(u_{j} \rho H\right)=\frac{\partial}{\partial x_{j}}\left(u_{i} \sigma_{i j}+\frac{C_{p} \mu}{P r} \frac{\partial T}{\partial x_{j}}\right)+S_{\rho E},
\end{gathered}
$$

where $\rho, u_{i}, p, H, E, \sigma_{i j}, T$ and $S$ are the density, velocity, pressure, enthalpy, energy, viscous stress, temperature and source terms respectively. $C_{p}$ is the specific heat constant, $\operatorname{Pr}$ is the Prandtl number and $\mu$ is the viscosity. The steady Reynolds-averaged Navier-Stokes (RANS) equations for compressible flow are:

$$
\begin{gathered}
\frac{\partial}{\partial x_{j}}\left(\bar{\rho} \widetilde{u_{j}}\right)=0 \\
\frac{\partial}{\partial x_{j}}\left(\bar{\rho} \widetilde{u_{j}} \widetilde{u_{i}}\right)=-\frac{\partial \bar{p}}{\partial x_{i}}+\frac{\partial\left(\overline{\sigma_{i j}}-\overline{\rho u_{i}^{\prime \prime} u_{j}^{\prime \prime}}\right)}{\partial x_{j}}, \\
\frac{\partial}{\partial x_{j}}\left(\bar{\rho} \widetilde{u_{j}} \widetilde{H}\right)=\frac{\partial}{\partial x_{j}}\left(\overline{\sigma_{i j}} \widetilde{u_{i}}+\frac{C_{p}}{P r} \overline{\frac{\partial T}{\partial x_{j}}}\right)+\frac{\partial}{\partial x_{j}}\left(-\overline{\rho u_{j}^{\prime \prime} u_{i}^{\prime \prime}} \widetilde{u_{i}}-\overline{\rho u_{j}^{\prime \prime} h^{\prime \prime}}-\frac{1}{2} \overline{\rho u_{j}^{\prime \prime} u_{i}^{\prime \prime} u_{i}^{\prime \prime}}+\overline{\sigma_{i j} u_{i}^{\prime \prime}}\right),
\end{gathered}
$$

where the overbar and tilde represent the Reynolds-averaged and Favre-averaged values respectively, and II represent the deviations from the Favre-averages. For a streamwise-periodic domain, all the streamwise mean gradient terms become zero in the RANS equations, which is not true for any spatially evolving flows. So to capture the spatially evolving behavior of the mean flow, one needs to add source terms corresponding to the streamwise mean gradients from Eq. 2 to the Navier-Stokes equations, Eq. 1. 


\section{A. Analytical Method}

For the rest of the paper, we will define $x$ and $z$ to be the streamwise and spanwise directions, respectively. The source terms $(S)$ appearing in the right hand side of Eq. 1 will read as:

$$
\begin{gathered}
S_{\rho}=-\frac{\partial}{\partial x}(\bar{\rho} \widetilde{u}), \\
S_{\rho u_{i}}=-\frac{\partial}{\partial x}\left(\bar{\rho} \widetilde{u} \widetilde{u_{i}}\right)-\frac{\partial \bar{p}}{\partial x} \delta_{i 1}+\frac{\partial\left(\overline{\sigma_{i 1}}-\overline{\rho u_{i}^{\prime \prime} u^{\prime \prime}}\right)}{\partial x}, \\
S_{\rho E}=-\frac{\partial}{\partial x}(\bar{\rho} \widetilde{u} \widetilde{H})+\frac{\partial}{\partial x}\left(\overline{\sigma_{i 1}} \widetilde{u_{i}}+\frac{C_{p}}{P r} \mu \frac{\partial T}{\partial x}\right)+\frac{\partial}{\partial x}\left(-\overline{\rho u^{\prime \prime} u_{i}^{\prime \prime}} \widetilde{u_{i}}-\overline{\rho u^{\prime \prime} h^{\prime \prime}}-\frac{1}{2} \overline{\rho u^{\prime \prime} u_{i}^{\prime \prime} u_{i}^{\prime \prime}}+\overline{\sigma_{i 1} u_{i}^{\prime \prime}}\right) .
\end{gathered}
$$

When the self-similar profiles of the state variables and their high-order moments are known analytically, then one can directly compute the source terms using Eq. 3 directly.

\section{B. Adjoint-Driven Optimization Method}

Analytic derivation of the source terms, (Eq. 3), is complicated. Further, the complete set of self-similar profiles required to evaluate the source terms are not always available. For example, self-similar profiles of high-order moments are very difficult to obtain. To address this, one can use an optimization technique to estimate the source terms to generate either a desired profile of the state variables, or any other desired engineering output (e.g. loss profiles, heat-transfer characteristic, noise profiles etc.). In the present work, the source terms are parameterized using Chebyshev polynomials. The quantity of interest (QoI), $\mathcal{J}$, of the minimization process is the error between the simulated and target mean profiles. An adjoint method is employed to evaluate the sensitivities of the objective to the multiple input parameters (the coefficients of the Chebyshev polynomials). We use the Sequential Least SQuares Programming algorithm from the SciPy optimization toolkit to drive the optimization procedure.

\section{Damped Adjoint-Driven Optimization Method}

The conventional adjoint method fails to estimate sensitivities of long-time averaged QoI, $\mathcal{J}$, to the input parameters for the turbulent flows due to the chaotic behavior. ${ }^{10}$ The adjoint variables grow exponentially for chaotic turbulent cases, resulting in essentially infinite sensitivity to the input parameters. Different methods exists in the literature ranging from computationally expensive least square shadowing method ${ }^{11,12}$ or space-split sensitivity method ${ }^{13}$ to stabilizing the adjoint equations by dissipation. ${ }^{14-16}$ In the present work, we have followed the adjoint equation stabilization through dissipation approach. As, the primal forward problem, Eq. 1, is solved using entropy variable, ${ }^{17}$ the corresponding adjoint equation for the Euler equation becomes:

$$
\int_{t} \int \frac{\partial \mathcal{J}}{\partial v_{i}}+\int_{t} \int_{\delta \Omega} \psi_{k} A_{k i}^{j} n_{j}+\int_{t} \int_{\Omega}\left\{-A_{k i}^{0} \frac{\partial \psi_{k}}{\partial t}-A_{k i}^{j} \frac{\partial \psi_{k}}{\partial x_{j}}\right\}=0
$$

where $A_{i k}^{0}=\frac{\partial q_{i}}{\partial v_{k}} . \quad A_{i k}^{j}=\frac{\partial F_{i}^{j}}{\partial v_{k}}=\frac{\partial F_{i}^{j}}{\partial q_{l}} \frac{\partial q_{l}}{\partial v_{k}}=\frac{\partial F_{i}^{j}}{\partial q_{l}} A_{l k}^{0}, \boldsymbol{q}, \boldsymbol{v}, \boldsymbol{\psi}$ are the conservative, entropy and adjoint variables and $\boldsymbol{F}$ is the inviscid fluxes. Note that the $\boldsymbol{A}^{\mathbf{0}}$ is a symmetric positive definite matrix and $\boldsymbol{A}^{\boldsymbol{j}_{\mathrm{S}}}$ are symmetric matrices. Now, the adjoint energy equation can be written as:

$$
\int_{t} \int_{\Omega} \frac{\partial\left(\psi_{i} A_{k i}^{0} \psi_{k}\right)}{\partial t}=\int_{t} \int_{\Omega} \boldsymbol{\psi}^{T} \boldsymbol{M} \boldsymbol{\psi}+\int_{t} \int_{\delta \Omega} \psi_{k} A_{k i}^{j} n_{j} \psi_{i}+2 \int_{t} \int \frac{\partial \mathcal{J}}{\partial v_{i}} \psi_{i}
$$

where $\boldsymbol{M}=\frac{\partial \boldsymbol{A}^{\mathbf{0}}}{\partial t}+\frac{\partial \boldsymbol{A}^{\boldsymbol{j}}}{\partial x_{j}}$ is the adjoint growth matrix. Since $\boldsymbol{A}^{\mathbf{0}}$ is a symmetric positive definite matrix, the left hand side of Eq. 5 is a positive number. Thus $\boldsymbol{M}$, which is also a symmetric matrix, primarily contributes to the growth of the adjoint variable. The boundary terms can also potentially contribute to the growth, but for now we are ignoring these contributions (as we are focused on periodic domains, adiabatic wall boundary conditions). The viscous terms in the Navier-Stokes equations act as dissipation terms in the 
adjoint energy equation, hence we do not include in the derivation. $\boldsymbol{M}$ is given by

$$
\left[\begin{array}{ccccc}
0 & \frac{\partial \sigma_{1 j}}{\partial x_{j}} & \frac{\partial \sigma_{2 j}}{\partial x_{j}} & \frac{\partial \sigma_{3 j}}{\partial x_{j}} & \frac{\partial u_{l} \sigma_{l j}}{\partial x_{j}}+\kappa\left(\frac{\partial T}{\partial x}+\frac{\partial T}{\partial y}+\frac{\partial T}{\partial z}\right) \\
\frac{\partial \sigma_{1 j}}{\partial x_{j}} & \frac{\mathcal{D}(\rho u u+p)}{\mathcal{D} t}+2 \frac{\partial p u}{\partial x} & \frac{\mathcal{D}(\rho u v)}{\mathcal{D} t}+\left(\frac{\partial p v}{\partial x}+\frac{\partial p u}{\partial y}\right) & \frac{\mathcal{D}(\rho u w)}{\mathcal{D} t}+\left(\frac{\partial p w}{\partial x}+\frac{\partial p u}{\partial z}\right) & \frac{\mathcal{D}(\rho u H)}{\mathcal{D} t}+\frac{\partial p\left(u^{2}+H\right)}{\partial x}+\frac{\partial p u v}{\partial y}+\frac{\partial p u w}{\partial z} \\
\frac{\partial \sigma_{2 j}}{\partial x_{j}} & \ldots & \frac{\mathcal{D}(\rho v v+p)}{\mathcal{D} t}+2 \frac{\partial p v}{\partial y} & \frac{\mathcal{D}(\rho v w)}{\mathcal{D} t}+\left(\frac{\partial p w}{\partial y}+\frac{\partial p v}{\partial z}\right) & \frac{\mathcal{D}(\rho v H)}{\mathcal{D} t}+\frac{\partial p u v}{\partial x}+\frac{\partial p\left(v^{2}+H\right)}{\partial y}+\frac{\partial p v w}{\partial z} \\
\frac{\partial \sigma_{3 j}}{\partial x_{j}} & \ldots & \ldots & \frac{\mathcal{D}(\rho w w+p)}{\mathcal{D} t}+2 \frac{\partial p w}{\partial z} & \frac{\mathcal{D}(\rho w H)}{\mathcal{D} t}+\frac{\partial p u w}{\partial x}+\frac{\partial p v w}{\partial y}+\frac{\partial p\left(w^{2}+H\right)}{\partial z} \\
0 & \ldots & \ldots & \ldots & \frac{\mathcal{D}\left(\rho H H-\frac{a^{2} p}{\gamma-1}\right)}{\mathcal{D} t}+2 \frac{\partial p u_{j} H}{\partial x_{j}}
\end{array}\right],
$$

where $\frac{\mathcal{D} X}{\mathcal{D} t}=\frac{\partial X}{\partial t}+\frac{\partial X u_{j}}{\partial x_{j}}$. The most negative eigenvalue of $\boldsymbol{M}$ contributes the most to the growth of the adjoint (note that the adjoint equations are solved backward in time). For the temporally evolving turbulent flows, we estimate $\boldsymbol{M}$ by averaging in the temporal and homogeneous directions. Then the contribution of the most negative eigenvalue of $\boldsymbol{M}$ is subtracted from the adjoint equation, Eq. 5 , as $f \boldsymbol{W}^{-} \boldsymbol{W}^{-{ }^{T}} \lambda^{-} \boldsymbol{\psi}$ to restrict the growth of the adjoint variable, where $\lambda^{-}$is the most negative eigenvalue and $\boldsymbol{W}^{-}$is the corresponding eigenvector and $f$ is a scale factor. Note that, after adding such a contribution to the adjoint equation, the adjoint variables become non-dual with respect to the primal variables. One can include back the dual of the adjoint damping term, $f \boldsymbol{W}^{-} \boldsymbol{W}^{-{ }^{T}} \lambda^{-} \boldsymbol{v}$, to the primal Navier-Stokes equation to make the damped adjoint sensitivity computation dual-consistent. The stabilized method will be further discussed in a future publication.

\section{Results}

The compressible Navier-Stokes equations are solved using a high-order, space-time discontinuous-Galerkin (DG) formulation. Details of the solver can be found at [17, and references therein]. The present solver also has an adjoint capability for unsteady and turbulent chaotic flows. ${ }^{11,17,18}$ In the present work, we will demonstrate producing a given instance of a spatially evolving velocity profile using the source term procedure utilizing the analytical and optimization approach. For the optimization method, the objective function is given by:

$$
\mathcal{J}=\overline{\left(u_{i}(x, y, z, t)-u_{i}^{t}(y)\right)^{2}}+\overline{\left(\rho(x, y, z, t)-\rho^{t}(y)\right)^{2}}+\overline{\left(p(x, y, z, t)-p^{t}(y)\right)^{2}},
$$

where superscript $t$ represents the target profiles. First we will evaluate the current analytical and optimization methods for a laminar case (laminar Blasius boundary-layer). Then we will use the methods for the chaotic turbulent cases (turbulent ZPG boundary-layer, turbulent jet profile).

\section{A. Laminar Blasius Boundary-Layer}

To verify the methodology, we start with a laminar boundary-layer $\left(R e_{\theta}=15, M a_{\infty}=0.1\right)$. Since we have used periodicity in the spanwise direction, $S_{\rho w}=0$. A no-slip adiabatic wall boundary condition is enforced at the wall $(y=0)$, and slip with penetration boundary condition at the "farfield", located at $y=2$, are used.

\section{Analytical method}

For a laminar profile, all of the RANS stress terms in the source term expressions (Eqn. 3) drop out. Then the source terms are further simplified by ignoring the viscous and thermal diffusion terms, following the incompressible laminar boundary-layer equations. The simplified source terms are then computed assuming a self-similar profile for a Blasius boundary-layer. Also, a wall-normal velocity at $y=2$ is used as the penetration velocity for the "farfield" boundary.

The resulting profiles, and the error from the target profiles, are plotted in Figs. 1 and 2, respectively. For the incompressible boundary-layer, the density and pressure profiles are independent of the wall-normal coordinates, while the present compressible simulation results have a small variation in the density and pressure. These produce a relatively large error with respect to the target profiles. The analytical source term method results in overprediction of streamwise velocity by about $20 \%$ at the farfield, although the wall-normal component recovers the target profile. 


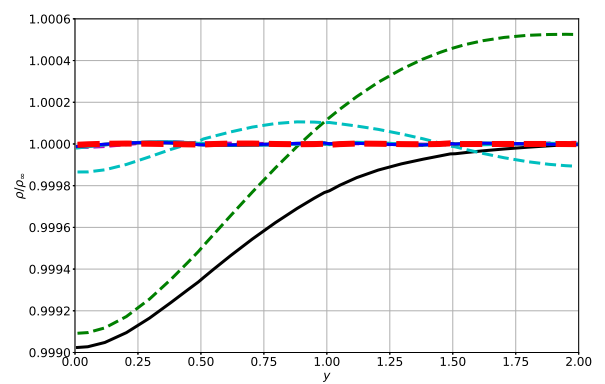

(a)

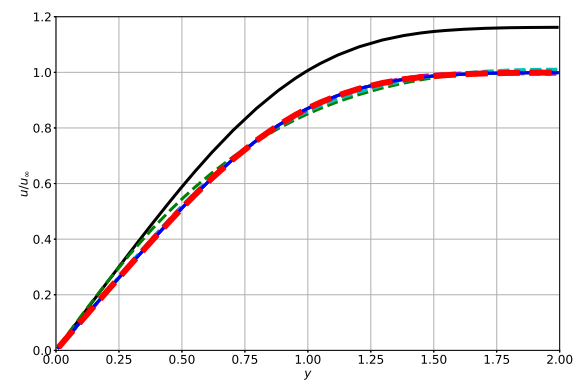

(c)

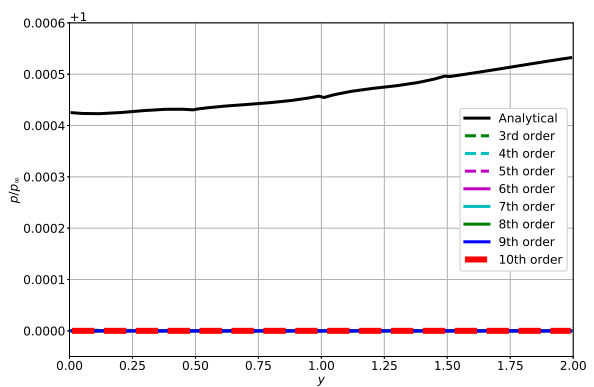

(b)

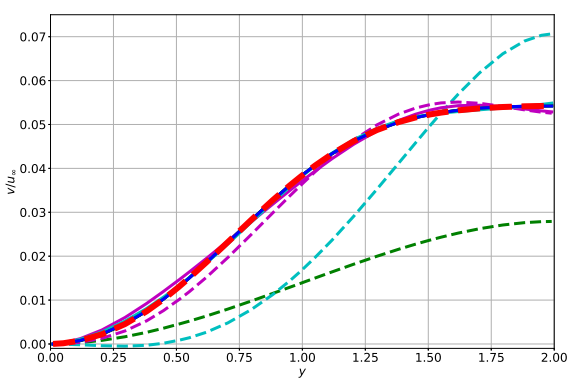

(d)

Figure 1: Simulated profiles of (a) density, (b) pressure, (c) streamwise and (d) wall-normal velocities for the laminar Blasius profile. 


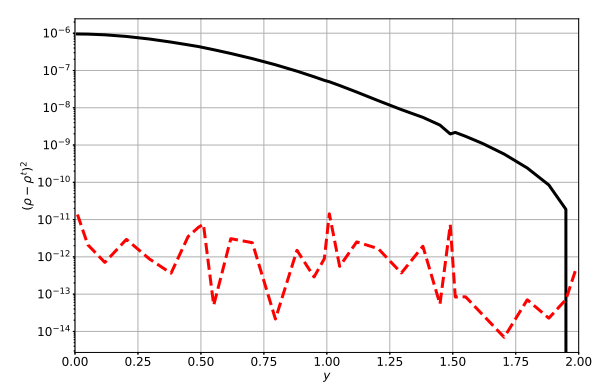

(a)

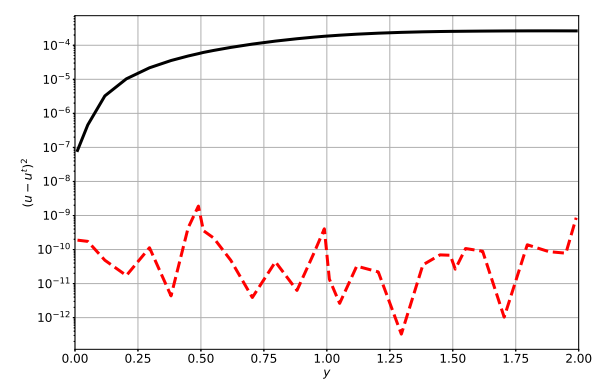

(c)

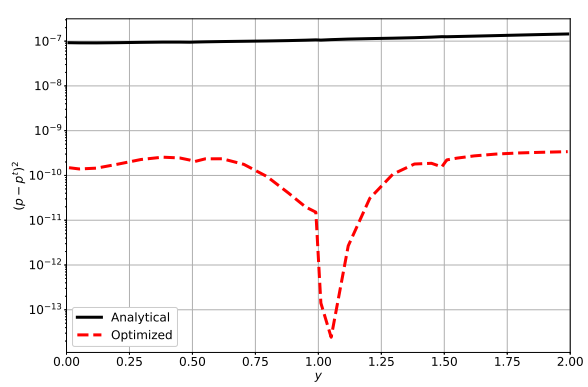

(b)

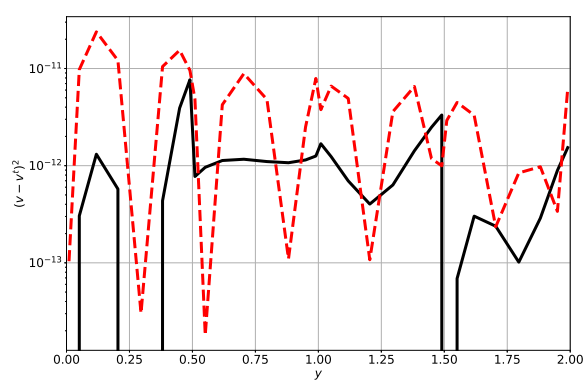

(d)

Figure 2: Contribution of different error terms from (a) density, (b) pressure, (c) streamwise and (d) wallnormal velocities to the $\mathcal{J}$ for the laminar Blasius profile.

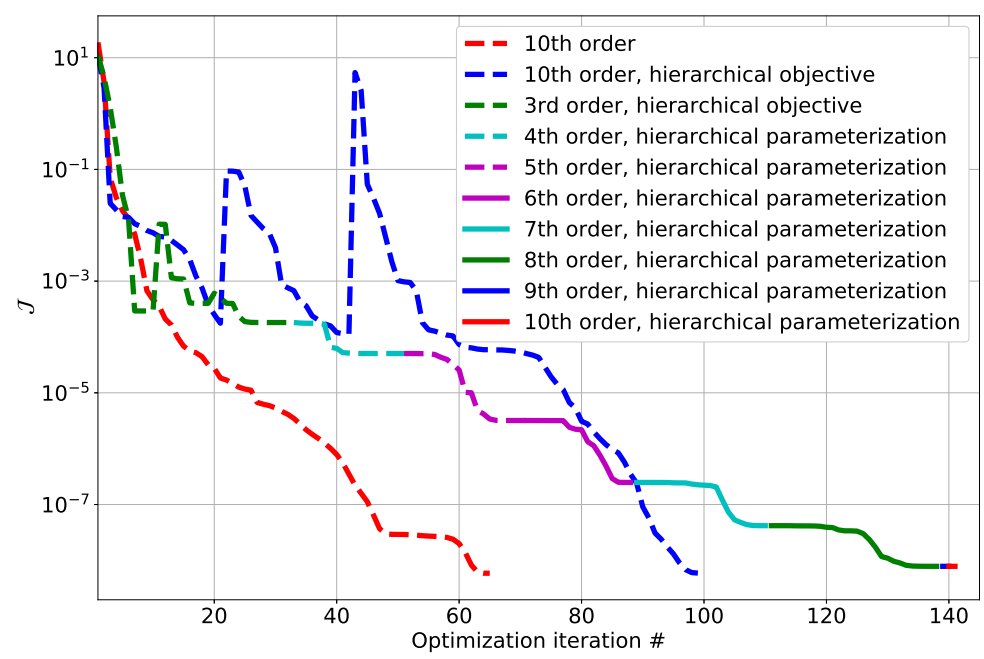

Figure 3: Evolution of the objective functional, $\mathcal{J}$, through the optimization process of laminar boundary layer. 


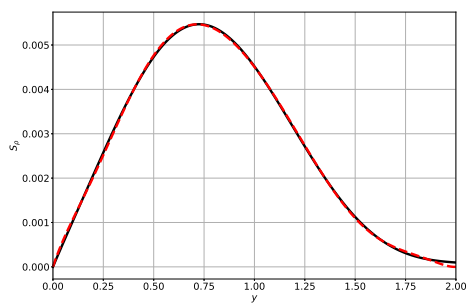

(a)

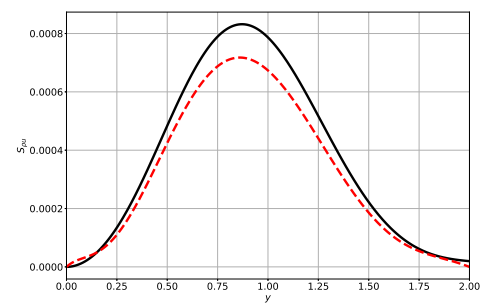

(b)

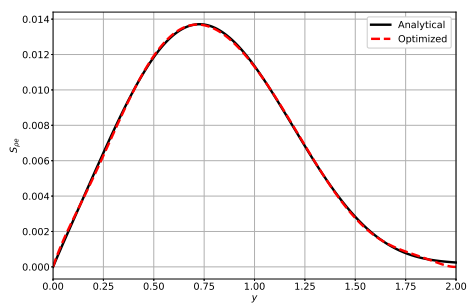

(c)

Figure 4: Comparison of the source terms for the (a) mass, (b) $x$-momentum and (c) energy equations between analytical and optimized method to simulate Blasius profile.

\section{Adjoint-driven optimization method}

Next, we have applied the adjoint-driven optimization method for simulating the Blasius profile. The source terms in the mass, $x$-momentum, and energy equations are parameterized using up to $10^{t h}$-order Chebyshev polynomials for each variables. We have dropped the $y$-momentum source term for the optimization method, as it is negligible. Also note that, as all the state variables are constant at the wall boundary for the incompressible boundary-layer, the source terms (Eq. 3) should be zero at the wall. The source terms should also be zero at the freestream. This is imposed by constraining first two Chebyshev coefficients such that source terms become zero at the wall and $y=2$. We have also used the penetration velocity at the farfield as an input parameter. Thus, the optimization method consists of total 25 parameters. The parameters associated with the Chebyshev polynomials are initiated with zero, and the penetration velocity is initialized to the analytical wall-normal velocity for the optimization method.

The objective (Eq. 7) drops to the machine zero (Fig. 3) and the sensitivity of the parameters also reduces by about 7 orders of magnitude over the optimization run. The resulting wall-normal profiles and the error are shown in Figs. 1 and 2. The optimization produces machine zero error in all the variables (Fig. 2) as we recover the target states.

Next, we compare the parameterized source terms with the analytical ones in Fig. 4. Estimated source terms for the mass (Fig. 4a) and energy (Fig. 4c) equations agree closely between the analytical and optimization method, with a small difference at the farfield location. These small discrepancies are mostly due to constraining the source terms at $y=2$. The $x$-momentum source term (Fig. $4 \mathrm{~b}$ ) from the optimization method is smaller compared to the analytical method for most of the $y$-locations. Note that we have assumed no viscous contribution for the analytical $x$-momentum source term (Eq. 3), and the difference in the curves of Fig. 4b mostly due to that. Also, the optimized penetration velocity at the farfield is about 0.998 times the wall-normal velocity at the farfield location.

The wall-normal velocity profiles are often not reported in experimental results. As such, we performed another optimization by removing the $y$-momentum error contribution from $\mathcal{J}$ to understand the optimization behavior. For this case, convergence of the optimization is poor, and the parameterized source terms are far from the analytical ones. This behavior can be clarified by looking into the mass equation, $\frac{\partial}{\partial y}(\rho v)+\frac{\partial}{\partial z}(\rho w)=S_{\rho}$. Thus, $S_{\rho}$ determines the profile of the wall-normal velocity. As we remove the $y$-momentum error contribution from the $\mathcal{J}$, the optimization problem becomes ill-posed, and it fails.

Note that the errors in $\rho, u, v$, and $p$ have different orders of magnitude. We modified the optimization procedure to include the different terms of the objective hierarchically, to drive down each error contributions. First, we perform the optimization for the $S_{\rho}$ and the penetration velocity at the farfield using the error contribution from $\rho$ and $v$. Then, we append the optimization to include the $S_{\rho u}$ using the error contribution for $u$. Finally, we add the optimization of $S_{\rho e}$ using the error contribution for $p$. The dashed blue line in the Fig. 3 shows the evolution of this hierarchical optimization process, and the peaks represent the inclusion of the different contributions of the objective. This optimization method also converges to the same optimized $\mathcal{J}$ as previously computed.

Next, we increase the Chebyshev polynomial order from 3 to 10 hierarchically. The resulting evolution of the optimization procedure is also shown in the Fig. 3. As, the polynomial order increases, the convergence 
of $\mathcal{J}$ improves. The resulting profiles of $\rho, u, v$ and $p$ are shown in the Figs. 1. The present hierarchical procedure reveals that at least $7^{\text {th }}$-order Chebyshev polynomials are required to drop the objective by 8 order for this case.

\section{Damped adjoint-driven optimization method}

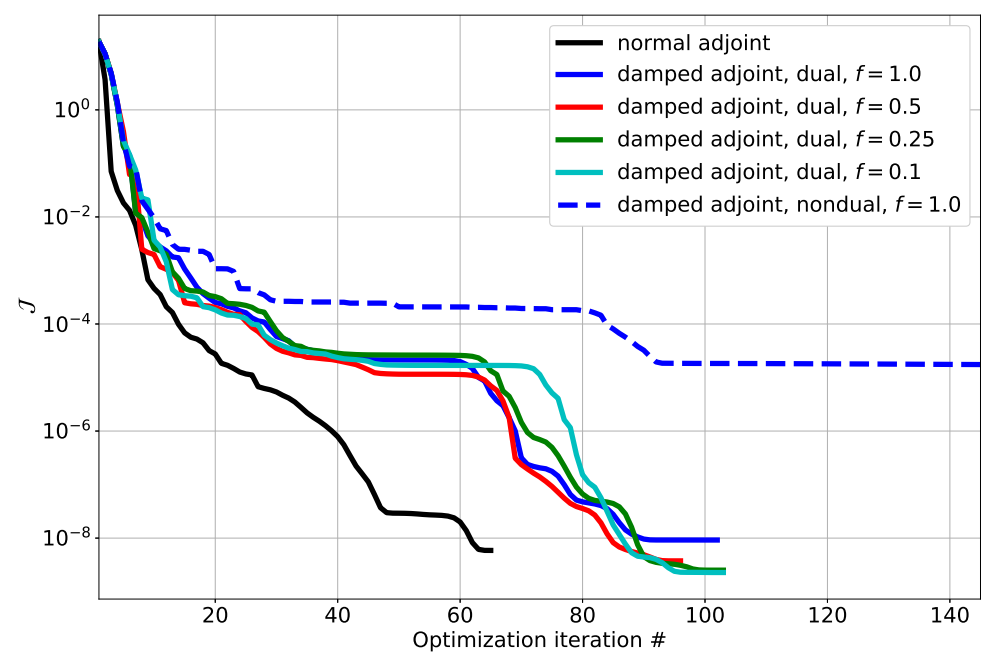

Figure 5: Comparison of the objective functional, $\mathcal{J}$ evolution for different different damp adjoint techniques of the laminar boundary layer.

Lastly, we have evaluated the damped adjoint technique for the laminar boundary layer optimization. Although the adjoint variables for the laminar boundary layer do not increase exponentially, $\boldsymbol{M}$ still has negative eigenvalues, though the dissipation for the laminar boundary layer is strong enough to restrict the exponential growth.

First, we only use the damping term in the adjoint equation for the optimization procedure. As mentioned earlier, this will violate dual consistency. The optimization process for this case stalls after dropping $\mathcal{J}$ by about 5 orders of magnitude (Fig. 5). Decreasing the scale factor, $f$, results in further drop of $\mathcal{J}$, but they all eventually stall. Now, when we add the dual terms for the adjoint damping to the primal, the optimization process converges to machine zero for all the scale factors. This behavior can be further explained by comparing the resulting source profiles for different $f$ with the normal adjoint (Fig. 6). Estimated source terms for the $x$ - and $y$-momentum equations vary strongly with $f$ as the source terms negate the additional dual terms in the primal equations, hence the optimization process for the damped adjoint non-dual case fails to converge. Source terms for the mass and energy equations are insensitive to the value of $f$. This is because the dominant terms in $\boldsymbol{M}$ are from the momentum equations. As $f$ decreases, the source terms moves toward the normal adjoint prediction, as expected. Note that, due to non-zero dual term in the $y$ momentum equation, we have also optimized for the source term for $y$-momentum equation for the damped adjoint cases, but not for the normal adjoint case. This results in a small difference in the optimized value of $\mathcal{J}$ between the normal and damped-dual adjoint.

\section{B. Turbulent Zero Pressure Gradient (ZPG) Boundary-Layer}

As the analytical and optimization methods are able to recover the laminar boundary-layer profile, we proceed to test the methods for the turbulent cases. First, we consider the turbulent ZPG boundary-layer profile $\left(R e_{\theta}=500\right.$ and $\left.M a_{\infty}=0.2\right)$. Similar to the laminar boundary-layer case, we have assumed $S_{\rho w}=0$ due to spanwise periodicity, and adiabatic no-slip boundary condition at the wall and slip with penetration boundary condition at the farfield. The computational domain spans $10 \delta \times 10 \delta \times 2.5 \delta$ where $\delta$ is the boundary- 


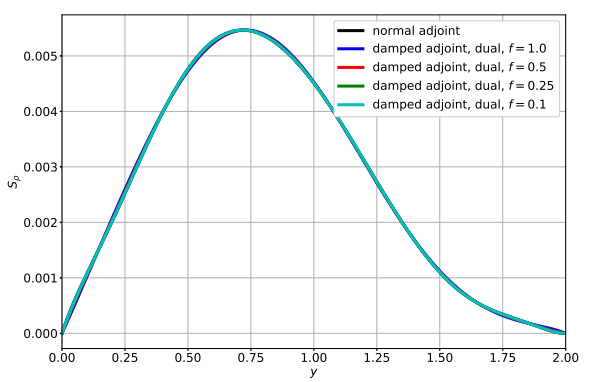

(a)

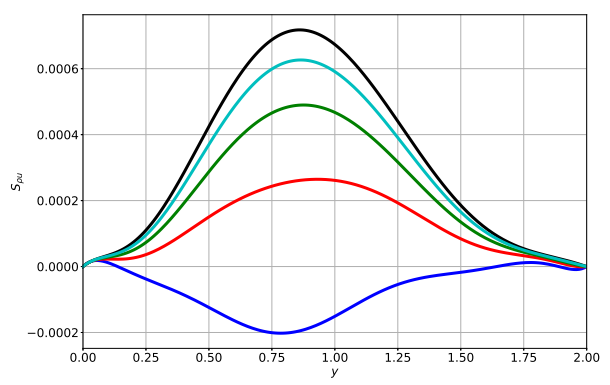

(c)

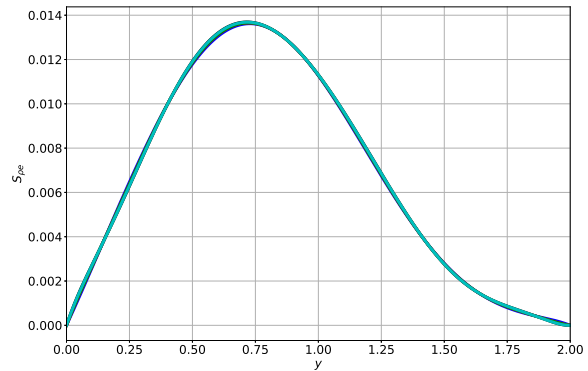

(b)

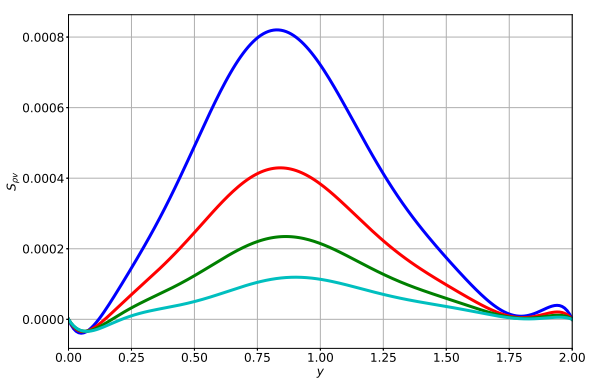

(d)

Figure 6: Comparison of the source terms for the (a) mass, (b) energy (c) $x$-momentum and (d ) $y$-momentum equations between different scale factor of damped adjoint technique. 
layer thickness, and is discretized using 16, 8, and 12 elements in the streamwise, wall-normal, and spanwise directions.

\section{Analytical method}

First, we examine the analytical procedure by approximating the source terms analytically. We have approximated the source terms in Eq. 3 by neglecting viscous and thermal diffusion terms, and also the higher-order moments of turbulent fluctuations. The streamwise mean gradient terms are estimated using the turbulent boundary-layer log profile with Cole's wake profile. ${ }^{19}$ The computational domain was discretized using either $4^{\text {th }}-(64 \times 32 \times 48$ degrees of freedom $)$ or $8^{\text {th }}$-order $(128 \times 64 \times 96$ degrees of freedom $)$ elements in the spatial directions and $4^{t h}$-order in the temporal direction. To generate turbulence, flow in the streamwise and spanwise periodic box is initiated with some perturbations. The simulations are then run long enough to reach a stationary state.

We compare our present results with Spalart's DNS results, ${ }^{9}$ and the target profiles in Fig. 7. The time sample for the statistics is accumulated for about $1050 \frac{\delta_{\nu}}{u_{\tau}}$, or $75 \frac{\delta}{U_{\infty}}$. Similar to the laminar boundary-layer, the wall-normal profiles of mean density (Fig. 7a) and pressure (Fig. 7b) reveal wall-normal variations. The mean density is slightly smaller than the target value and mean pressure is higher than the target value. The log-layer behavior of the mean streamwise velocity is well captured by the $8^{\text {th }}$-order solution compared to the $4^{t h}$-order solution, although it is underestimated slightly at the freestream (Fig. 7c). The wall normal velocity component agrees well with the target profile for both the discretization (Fig. 7d).

The profiles for turbulent fluctuations compare well with the Spalart's DNS data, except at the edge of the boundary layer (Fig. 8) for $8^{\text {th }}$-order simulation. The $4^{\text {th }}$-order (under-resolved) simulation resulted overestimation of the near wall streamwise turbulent intensity (Fig. 8a). The present simulation results show a slower decay of the turbulent fluctuations for $y>0.6 \delta$ compared to the DNS, resulting in a higher value at the boundary-layer edge for both the spatial discretization. We believe that these observed discrepancies are due to omitting the high-order moment contributions in the source term calculations.

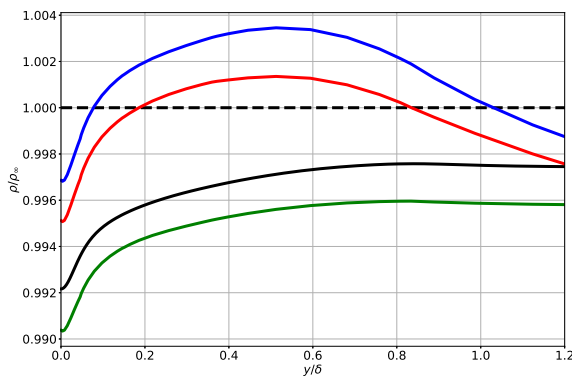

(a)

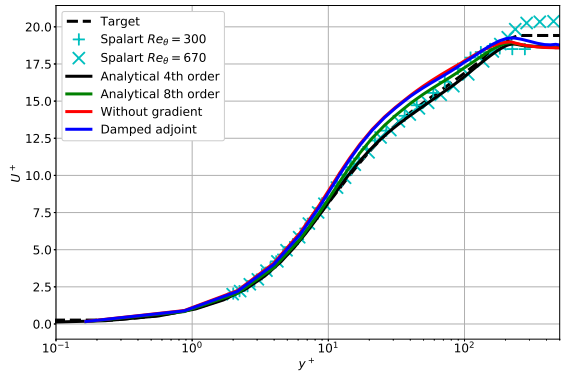

(c)

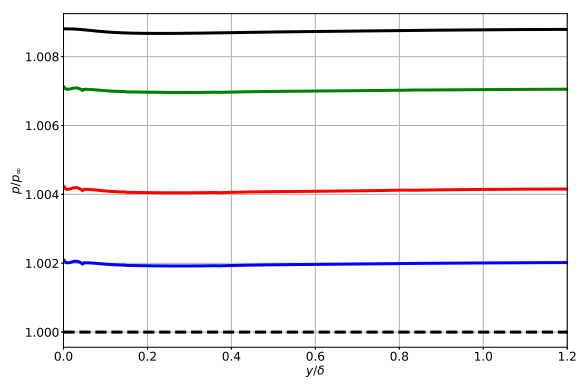

(b)

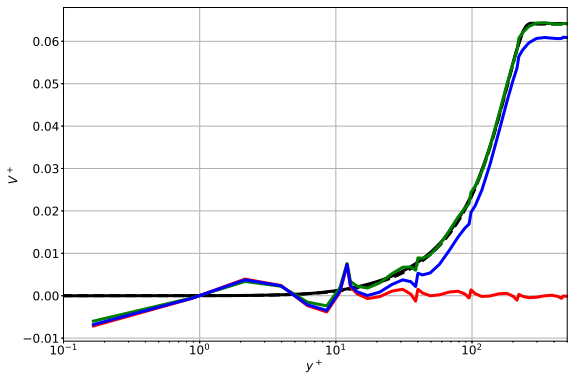

(d)

Figure 7: Wall-normal profiles of (a) mean density, (b) mean pressure, (c) mean streamwise velocity, and (d) mean wall normal velocity components for the turbulent ZPG boundary-layer. 


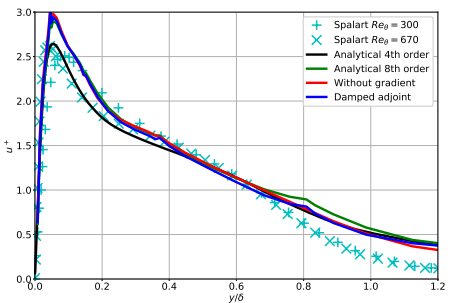

(a)

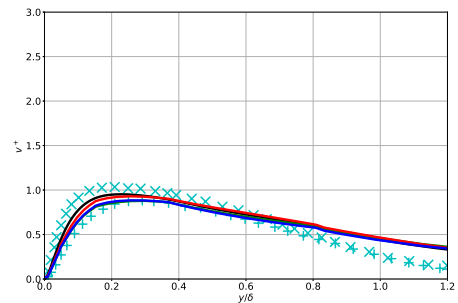

(b)

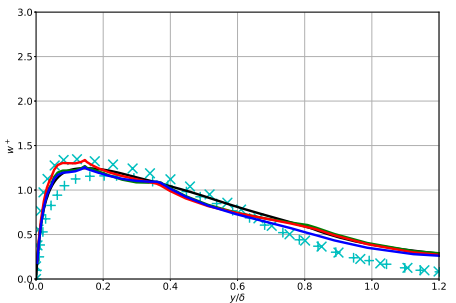

(c)

Figure 8: Wall-normal profiles of the (a) streamwise, (b) wall-normal, and (c) spanwise turbulent velocity components for the turbulent ZPG boundary-layer.

\section{Adjoint-driven optimization method}

To estimate the source terms using the optimization method, one has to run the forward problem long enough $\left(t_{s t}\right)$ to reach a stationary state, and then perform averaging long enough $\left(t_{\text {ave }}\right)$ to achieve statistical stationarity. For this example, we have used $4^{t h}$-order elements both in the spatial and temporal directions to reduce the computational cost. For the turbulent boundary layer simulations, we have used Chebyshev polynomials to parameterize the source terms in the mass, $x$-momentum, and energy equations, and the penetration velocity at the farfiled boundary as the optimization parameters. The first two Chebyshev coefficients are constrained to result zero source terms at the wall and the edge of the boundary layer. The Chebyshev polynomial coefficients are initiated with some random values.

First, we study the behavior of the adjoint variables for the chaotic turbulent flow. Figure 9 shows the temporal evolution of the $x$-momentum adjoint in a $x-y$ plane. Note that the adjoint goes backwards in time, hence time is reported as negative. The adjoint variable is non-zero close to the wall where turbulence is getting generated for small negative time. The magnitude of the adjoint continues to grow as it is solved backwards, and it takes large values over the whole computational domain. The exponential growth happens because of inherent chaotic nature of turbulent flow. Similar observations for the chaotic adjoint are also made by Wang \& Gao, ${ }^{10}$ Blonigan, ${ }^{11} \mathrm{Ni},{ }^{12}$ and Chandramoorthy et al. ${ }^{13}$

One of the proposed methods to reduce the exponential growth of the adjoint variables is to use smaller windowing function on $\mathcal{J}{ }^{20}$ In the present work we have used a square window. Figure 10 shows the temporal evolution of the sum of $L_{2}$-norm of the adjoint variables for different windows. Exponential growth of the adjoint variable is evident for all the cases considered. Neither running the forward problem for a shorter time, nor performing averaging over a shorter window, will mitigate the exponential growth. Thus any parameter sensitivity $\left(\frac{\partial \mathcal{J}}{\partial \alpha}=\frac{\partial \mathcal{R}}{\partial \alpha}^{T} \psi\right)$ is overwhelmed by the magnitude of the adjoint, making it unusable for the optimization problem.

\section{Damped adjoint-driven optimization method}

To address the exponential growth of the chaotic adjoint, we have employed the damped adjoint technique described in the Section 2. When we employ the damping terms in the adjoint equations only, i.e. a dual-inconsistent formulation, growth of the adjoint norms reduces (Fig. 10). A higher value of the scaling factor, $f$, results in the least amount of the growth. As noted in the laminar boundary layer case, the dualinconsistent formulation results in stalling of the optimization procedure. Employing the dual-consistent adjoint damping method for the turbulent case fails to stop the exponential growth of the adjoint variables. As we add dual of the adjoint damping term to the primal Navier-Stokes equations, the base primal solution gets modified, hence $\boldsymbol{M}$ changes. One alternative is to perform multiple iterations of $\boldsymbol{M}$ estimations in a recursive way, but it is not clear that such a cyclic procedure will converge. We don't observe this behavior for the laminar flow case, as the normal adjoint itself does not show any exponential growth.

Next, we proceed with the optimization method using the dual-inconsistent damped adjoint technique with $f=1.0$. We have also performed a "gradient free" optimization using Constrained Optimization By Linear Approximation (COBYLA) algorithm. For turbulent optimizations, we used up to $3^{\text {rd }}$-order Cheby- 


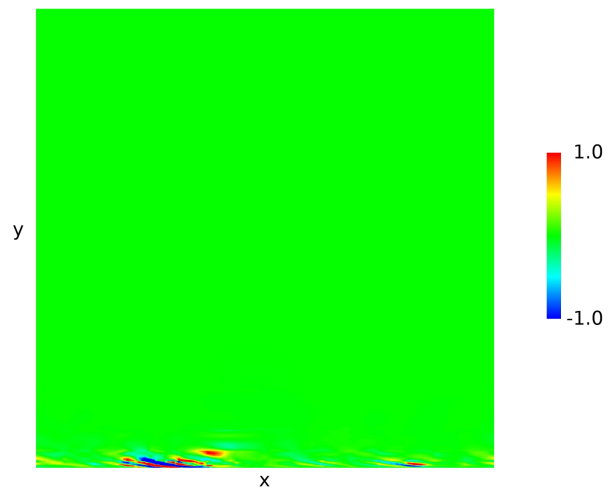

(a)

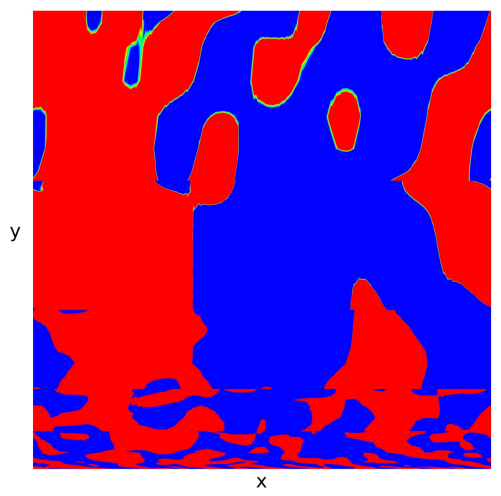

(c)

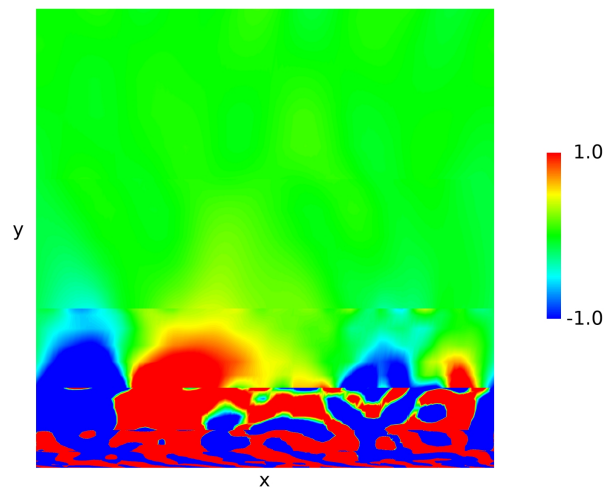

(b)

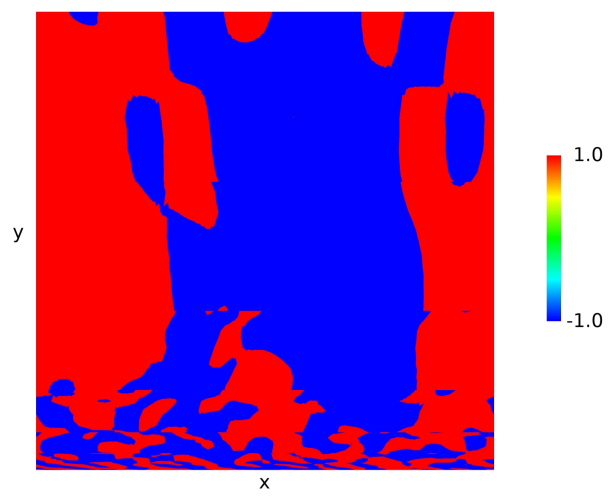

(d)

Figure 9: Contour of $x$-momentum adjoint variable in a $x-y$ plane at $t=-38 \frac{\delta}{U_{\infty}}$ (Fig. a), $-77 \frac{\delta}{U_{\infty}}$ (Fig. b), $-116 \frac{\delta}{U_{\infty}}$ (Fig. c), and $-155 \frac{\delta}{U_{\infty}}$ (Fig. d) for the $t_{s t}=130 \frac{\delta}{U_{\infty}}$ and $t_{a v e}=30 \frac{\delta}{U_{\infty}}$ case. 


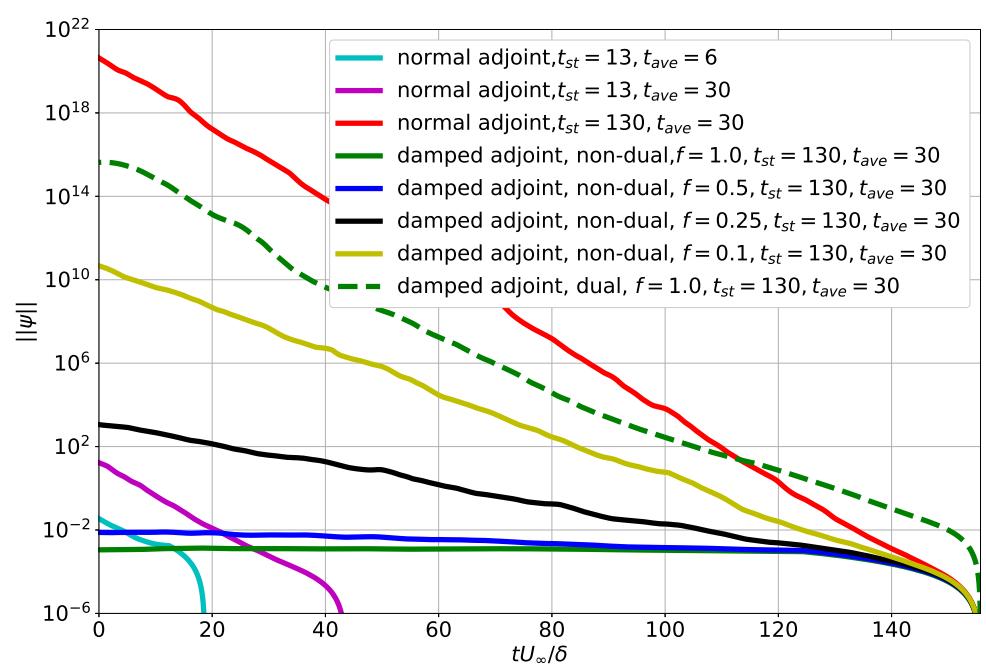

Figure 10: Temporal evolution of the sum of $L 2$ norm of adjoint variables for different $t_{s t}, t_{\text {ave }}$, and different adjoint techniques. $t$ is normalized by $\frac{\delta}{U_{\infty}}$.

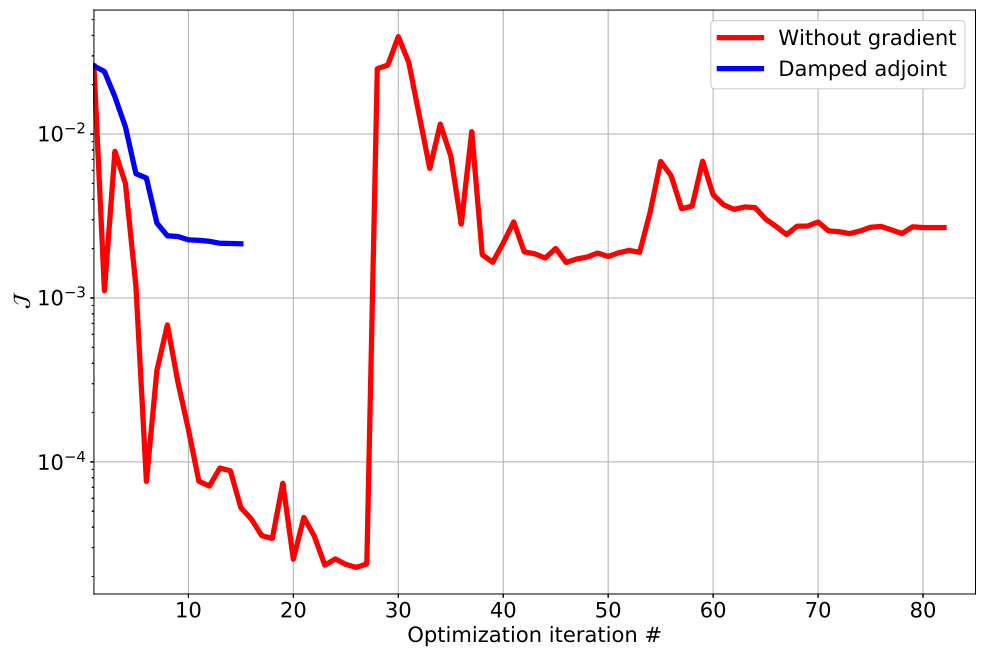

Figure 11: Evolution of the objective functional, $\mathcal{J}$, through the optimization process using $t_{s t}=130 \frac{\delta}{U_{\infty}}$ and $t_{\text {ave }}=30 \frac{\delta}{U_{\infty}}$ case. 
shev polynomials. Both the optimization procedures stalled after dropping the $\mathcal{J}$ by an order of magnitude (Fig. 11). Peaks in the evolution of $\mathcal{J}$ for the "gradient free" optimization are due to appending different objective contributions hierarchically, as prescribed in the laminar boundary layer case. The resulting mean and turbulent wall normal profiles are shown in the Figs. 7 and 8. The optimization methods reduce the error in the mean density and pressure profiles compared to the analytical method (Fig 7a, and 7b). The mean streamwise velocity profiles from the optimization procedures agree well with the $4^{t h}$-order analytical solution. Underestimation of the freestream streamwise velocity still persists (Fig. 7c). Although, both the optimization technique result in similar final $\mathcal{J}$ (Fig. 11), the wall-normal velocity profile is closer to the target state from the optimization using the damped adjoint technique compared to the "gradient free" optimization (Fig. 7d). Note that the wall-normal velocity is orders of magnitude smaller compared to the other state variables. Weighting the wall-normal velocity error higher for the "gradient free" optimization did not improve the results. The estimated turbulent velocity profiles from the optimization methods are similar to the $4^{\text {th }}$-order analytical solutions (Fig. 8). Increase in the Chebyshev polynomial order up to $5^{\text {th }}$, or increase in the $t_{s t}+t_{\text {ave }}$ do not help to reduce $\mathcal{J}$ further.

Note that we don't constrain the high-order moments of the turbulent velocities in the current optimization procedure, as these are not typically reported in the experiment results.

In the present work, we have only considered SLSQP and COBYLA algorithm. Bayesian optimization is an attractive alternative for noisy and computationally expensive objective function. Bayesian optimization with the gradient information is still an active field of research. Talnikar ${ }^{21}$ have used Bayesian optimization with the gradient information to design a high-pressure turbine trailing edge. In the future work, we will evaluate the performance of the Bayesian optimization for the current optimization problem.

\section{Turbulent Jet Profile}

Next, we test the methods to generate an experimentally observed turbulent jet profile. We have considered a wall-jet configuration from a fundamental trailing-edge cooling slot for high-pressure turbine airfoils. Experimental data is obtained from Kacker \& Whitelaw. ${ }^{22,23}$ Simulating the full geometry for the walljet configuration ${ }^{22}$ makes the scale-resolving simulation computationally expensive. Also, only the mean streamwise velocity at the wall-jet slot exit was measured in the experiment, and the flow asymmetry was noted. We would like to generate the asymmetric mean streamwise velocity at the wall-jet slot exit with turbulent fluctuations as an inlet condition for the main wall-jet computation.

\section{Analytical method}

Mean streamwise gradient informations, required to analytically estimate the source terms (Eqn. 3), were not reported in the experiment. So we have assumed a fully developed turbulent channel profile at the upstream of jet slot exit, as a very crude approximation. The turbulent channel flow parameters are estimated using bulk flow condition, and using friction velocity correlations. ${ }^{19}$ Then, only the $x$-momentum source term, $S_{\rho u}=\frac{2 \tau_{w}}{y_{c}}$ where $y_{c}$ is the wall-jet slot thickness and $\tau_{w}$ is the wall friction, is applied. The turbulent channel profile is computed using $8 \times 8 \times 10$ elements in a $6 y_{c} \times y_{c} \times 6 y_{c}$ streamwise and spanwise periodic box. Adiabatic wall boundary conditions are used at both the walls. In spatial direction both $4^{\text {th }}-(32 \times 32 \times 40$ degrees of freedom) and $8^{\text {th }}$-order $(64 \times 64 \times 80$ degrees of freedom $)$ discretizations, and in temporal directions $4^{\text {th }}$-order discretization are used to simulate the flow.

The resulting mean streamwise velocity profile is plotted in Fig. 12. The $4^{t h}$-order simulation results in a resolution of $x^{+} \approx 60, z^{+} \approx 60$, and $y^{+} \approx 4$ at the walls. Hence, the resulting streamwise velocity profile does not show a log-law behavior, and it also overpredicts the centerline velocity, as expected. The $8^{\text {th }}$-order simulation results in $x^{+} \approx 30, z^{+} \approx 30$, and $y^{+} \approx 2$ at the walls. Thus, the resulting profile captures the $\log$ law reasonably well. Note that an asymmetric velocity profile is noticed in the experimental measurement. This asymmetry can not be captured by the fully developed turbulent channel profile assumption.

\section{Damped adjoint-driven optimization method}

Next, we use the optimization method to capture the flow asymmetry as observed. The normal adjoint technique fails as the flow is turbulent, and we proceed with the damped adjoint method. We have also performed a "gradient free" optimization for comparison. For this case, we have used the first two Chebyshev polynomial coefficients for the mass, $x$-momentum, and energy source term parameterizations. Since, the 


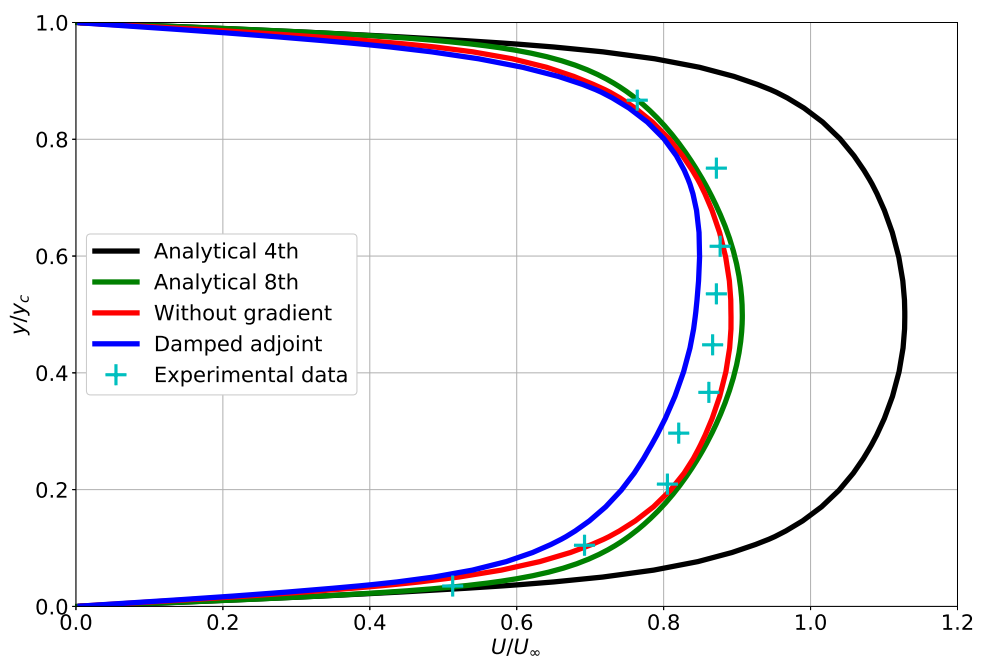

Figure 12: Wall-normal profiles of the mean streamwise velocity for the turbulent jet.

wall-normal velocity profile was not reported in the experiment, we did not include wall-normal velocity error in the definition of $\mathcal{J}$. Also, the bulk density and the wall pressure are used to compute the density and the pressure error in $\mathcal{J}$. For the optimization technique, $4^{\text {th }}$-order discretizations in space and time are used. The turbulent flow is initialized with the solution from the analytical method, and the optimization parameters are initialized to zero for the optimization procedure.

For both the optimization procedures, using damped adjoint and "gradient free" optimization, the $\mathcal{J}$ is dropped by about one order (Fig. 13). Resulting mean streamwise velocity profiles are also plotted in Fig. 12. The "gradient free" optimization fails to capture the flow asymmetry, whereas the optimization with damped adjoint captures it. Although the centerline velocity is underestimated by the optimization with damped adjoint compared to the "gradient free" optimization.

Unlike the laminar flow case, the optimization process does not drive $\mathcal{J}$ to machine zero for the turbulent flow cases. The optimization procedures using adjoint damped technique, and "gradient free" result in different answers. The observed stalling of the optimization procedure may be due to the non-linearity of the problem, or inaccurate parameter sensitivity estimation as we use non-dual adjoint technique (for laminar flow case, non-dual adjoint technique stalled, Fig. 5), or under-constraining the optimization problem by not considering high-order moments of turbulent quantities. Although the final results using the optimization method are still superior the other options for the problem.

\section{Conclusion}

In this work we have demonstrated a novel technique to generate inflow turbulent profiles using source terms in the Navier-Stokes equations in a streamwise- and spanwise-periodic domain. To estimate the source terms using analytical expressions is very involved and limited to self-similar flows. But, most of the experimental observations are far from any self-similar behavior. For any generic turbulent profiles, we have formulated an optimization problem by parameterizing the source terms. Sensitivities to the parameters of the source terms are computed using an adjoint technique. The present technique is then tested on a laminar Blasius profile, turbulent ZPG boundary-layer profile, and a turbulent jet profile.

The optimization method reduces the profile errors to machine zero for the laminar Blasius profile case. For the turbulent flow cases, traditional adjoint method fails to estimate the sensitivities due to chaotic turbulent flow behavior. We have used an adjoint damping method to restrict the exponential growth. Adding the damping terms in the adjoint equations makes the problem dual-inconsistent, and the optimization method stalls for the laminar case. Adding the dual of the damping term to the primal makes the problem 


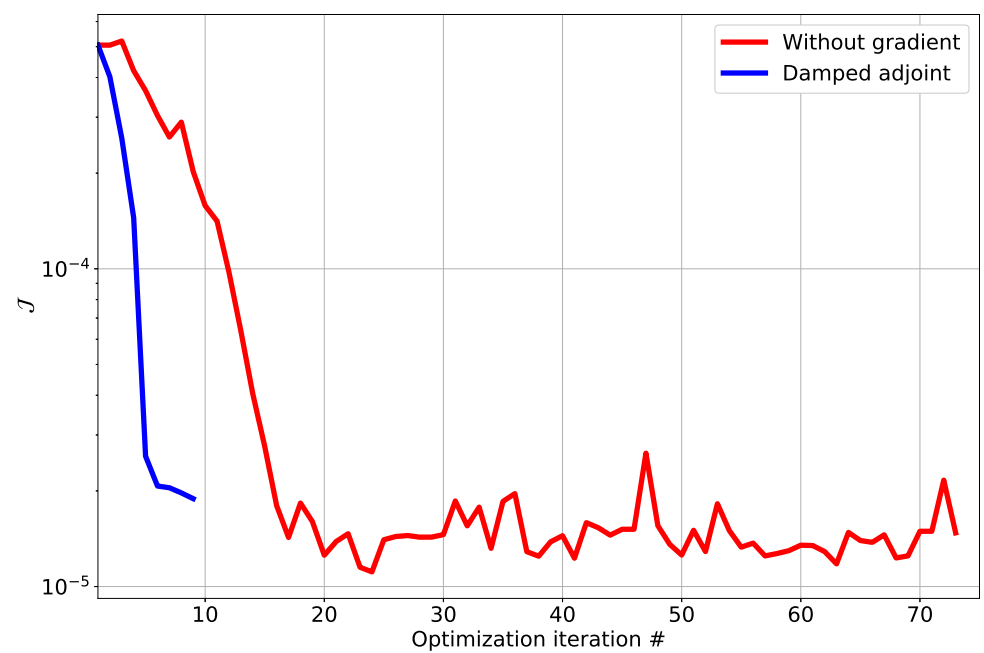

Figure 13: Evolution of the $\mathcal{J}$ through the optimization process for jet profiles.

dual consistent, and the optimization method reduces the error to machine zero. However for the turbulent cases, dual-consistent adjoint damping technique fails to eliminate the exponential growth. The optimization procedure using the dual-inconsistent adjoint damping technique drops the objective by about one order of magnitude for the turbulent cases and then stalls. This may be due to the non-linearity of the problem, or inaccurate sensitivity predictions (non-dual adjoint), or not constraining the high-order moments of the turbulent flow variables. The least-square shadowing technique ${ }^{11,12}$ or space-split sensitivity technique ${ }^{13}$ can be employed to estimate the sensitivities accurately, but the procedure would be computationally very expensive.

For the turbulent cases, we have also tried the "gradient free" optimization technique. But the profiles from the damped adjoint technique are closer to the target profiles compared to the "gradient free" optimization, e.g. wall-normal velocity profile for turbulent ZPG boundary-layer case and flow asymmetry in the turbulent jet case. Other optimization approaches, e.g. Bayesian optimization, will also be evaluated in the future. Although the optimization methods do not converge $\mathcal{J}$ to machine zero for the turbulent cases, the non self-similar flow features (e.g. flow asymmetry in the turbulent jet case) are captured satisfactorily.

Most of the experimental observations can be considered as a perturbation from either the ZPG boundary layer, or channel flow, as they originate from either boundary-layer or duct flow configurations. Our goal is to create a database of the self-similar profiles, which can then be modified using the optimization method to capture the experimental observations. The turbulent jet flow case has successfully demonstrated feasibility of such an approach.

\section{Acknowledgments}

Funding was provided by the Advanced Air Transport Technology Project in the NASA Advanced Air Vehicles Program, and the Transformational Tools and Technologies Project in the NASA Transformative Aeronautics Concepts Program. Computing resources were provided by the NASA Advanced Supercomputing facility at NASA Ames Research Center.

\section{References}

\footnotetext{
${ }^{1}$ Garai, A., Diosady, L. T., Murman, S. M., and Madavan, N. K., "Development of a perfectly matched layer technique for a Discontinuous-Galerkin spectral-element method," AIAA Paper 2016-1338, 2016.

${ }^{2}$ Rai, M. M. and Moin, P., "Direct numerical simulation of transition and turbulence in a spatially evolving boundary layer," Journal of Computational Physics, Vol. 109, 1993, pp. 169-192.
} 
${ }^{3}$ Fasel, H. F., Rist, U., and Konzelmann, U., "Numerical investigation of the three-dimensional development in boundarylayer transition," AIAA Journal, Vol. 28, No. 1, 1990, pp. 29-37.

${ }^{4}$ Pirozzoli, S., Grasso, F., and Gatski, T. B., "Direct numerical simulation and analysis of a spatially evolving supersonic turbulent boundary layer at $\mathrm{M}=2.25$," Physics of Fluids, Vol. 16, No. 3, 2004, pp. 530.

${ }^{5}$ Jarrin, N., Benhamadouche, S., Laurence, D., and Prosser, R., "A synthetic-eddy-method for generating inflow conditions for large-eddy simulations," International Journal of Heat and Fluid Flow, Vol. 27, 2006, pp. 585-593.

${ }^{6}$ Garai, A., Diosady, L. T., Murman, S. M., and Madavan, N. K., "Scale-resolving simulations of bypass transition in a high-pressure turbine cascade using a spectral-element Discontinuous-Galerkin method," Journal of Turbomachinery, Vol. 140, No. 3, 2017, pp. 031004.

${ }^{7}$ Lund, T. S., "Generation of turbulent inflow data for spatially-developing boundary layer simulations," Journal of Computational Physics, Vol. 140, 1998, pp. 233-258.

${ }^{8} \mathrm{Xu}$, S. and Pino Martin, M., "Assessment of inflow boundary conditions for compressible turbulent boundary layers," Physics of Fluids, Vol. 16, 2004, pp. 2623-2639.

${ }^{9}$ Spalart, P. R., "Direct simulation of turbulent boundary layer up to $\operatorname{Re}_{\theta}=1410$," Journal of Fluid Mechanics, Vol. 187, 1988, pp. 61-98.

${ }^{10}$ Wang, Q. and Gao, J.-H., "The drag-adjoint field of a circular cylinder wake at Reynolds numbers 20, 100 and 500," Journal of Fluid Mechanics, Vol. 730, 2013, pp. 145-161.

${ }^{11}$ Blonigan, P. J., "Adjoint sensitivity analysis of chaotic dynamical systems with non-intrusive least squares shadowing," Journal of Computational Physics, Vol. 348, 2017, pp. 803-826.

${ }^{12} \mathrm{Ni}$, A., "Hyperbolicity, shadowing directions and sensitivity analysis of a turbulent three-dimensional flow," Journal of Fluid Mechanics, Vol. 863, 2019, pp. 644-669.

${ }^{13}$ Chandramoorthy, N., Wang, Z. N., Wang, Q., and Tucker, P., "Toward computing sensitivities of average quantities in turbulent flows," The 2018 Summer Program, Center for Turbulence Research, Stanford, 2018, pp. 195-204.

${ }^{14}$ Talnikar, C., Wang, Q., and Laskowski, G. M., "Unsteady adjoint of pressure loss for a fundamental transonic vane," Journal of Turbomachinery, Vol. 139, 2017, pp. 031001.

${ }^{15}$ Ashley, A., Crean, J., and Hicken, J., "Towards aerodynamic shape optimization of unsteady turbulent flows," AIAA Paper 2019-0168, 2019.

${ }^{16}$ Bhatia, M. and Taoudi, L., "Assessment of stabilized solution approach for high-dimensional chaotic systems," AIAA Paper 2019-0170, 2019.

${ }^{17}$ Carton de Wiart, C., Diosady, L. T., Garai, A., Burgess, N., Blonigan, P., Ekelschot, D., and Murman, S. M., "Design of a modular monolithic impicit solver for multi-physics applications," AIAA Paper 2018-1400, 2018.

${ }^{18}$ Ceze, M., Diosady, L. T., and Murman, S. M., "Development of a high-order space-time matrix-free adjoint solver," AIAA Paper 2016-0833, 2016.

${ }^{19}$ Pope, S. B., Turbulent flows, Cambridge University Press, Cambridge, UK, 2000.

${ }^{20}$ Krakos, J., Unsteady adjoint analysis for output sensitivity and Mesh adaptation, Ph.D. thesis, Massachusetts Institute of Technology, 2012.

${ }^{21}$ Talnikar, C., Viscosity stabilized adjoint method for unsteady compressible Navier-Stokes equations, Ph.D. thesis, Massachusetts Institute of Technology, 2018.

${ }^{22}$ Kacker, S. C. and Whitelaw, J. H., "Some properties of the two-dimensional turbulent wall jet in a moving stream," Journal of Applied Mechanics, Vol. 35, 1968, pp. 641-651.

${ }^{23}$ Kacker, S. C. and Whitelaw, J. H., "The turbulence characteristics of two-dimensional wall-jet and wall-wake flows," Journal of Applied Mechanics, Vol. 38, 1971, pp. 239-252. 